\title{
The overall uncertainty of single-epoch virial black hole mass estimates and its implication to the $\mathrm{M}_{\mathrm{BH}}=\sigma_{*}$ relation
}

\author{
Jong-Hak Woo* and Daeseong Park \\ Seoul National University \\ E-mail: woo@astro.snu.ac.kr,pds2001@astro.snu.ac.kr
}

\begin{abstract}
Black hole mass is one of the key parameters in understanding the black hole-galaxy coevolution. Most black hole masses are determined from single-epoch spectra based on the virial assumption of the broad-line region (BLR) and the empirical relation of the BLR size with AGN continuum luminosity. Although understanding the uncertainty of single-epoch mass estimates is crucial, the overall uncertainty of this method is not well known. Using the homogeneous high-quality multiepoch data from the Lick AGN Monitoring Project, we investigate the uncertainties of singleepoch virial black hole masses by comparing measurements from single-epoch, mean, and rms spectra. The random errors due to the variability are $\sim 5 \%$ for line velocity of the $\mathrm{H} \beta$, and $\sim 13 \%$ for AGN continuum luminosity at $\mathrm{L}_{5100}$, while the random error due to the combined variability is $10-30 \%$. These results suggest that single-epoch masses are consistent within $\sim 30 \%$ uncertainty. Combining random errors due to line width and luminosity variability with the uncertainties of the virial factor and the size-luminosity relation, we estimate the overall uncertainty of singleepoch mass estimates as a factor of $\sim 3$. We find that virial products estimated from single-epoch spectra are systematically larger than those estimated from rms spectra, particularly for objects with narrow-lines (FWHM $<2000 \mathrm{~km} / \mathrm{s}$ ). We dicuss the implication of the systematic difference of $\mathrm{H} \beta$ line width in studying black hole-galaxy coevolution.
\end{abstract}

Narrow-Line Seyfert 1 Galaxies and their place in the Universe - NLS1,

April 04-06, 2011

Milan Italy

\footnotetext{
* Speaker.
} 


\section{Introduction}

Since black hole mass (BHM) is one of the fundamental properties of AGN, it is crucial to determine BHM in understanding AGN physics and black hole-galaxy coevolution (e.g., Davis et al. 2008; Woo \& Urry 2002; Kollmeier et al. 2006). The so-called single-epoch (SE) method has been developed based on the reverberation studies, utilizing the empirical relationship between the size of the BLR and the AGN continuum luminosity $\left(R_{\mathrm{BLR}} \propto L^{0.5}\right.$; Kaspi et al. 2000, 2005; Bentz et al. 2006, 2009a). Since BHM can be determined from single-epoch spectra, this method has been widely used for many AGN samples from large spectroscopic surveys (e.g., Shen et al 2008). The SE method is best-calibrated with the $\mathrm{H} \beta$ line and AGN optical luminosity as:

$$
M_{\mathrm{BH}} \propto f V^{2} L^{\sim 0.5}
$$

where $f$ is a virial factor which depends on the geometry and the kinematics of the BLR, $V$ is the line-of-sight velocity of BLR gas, measured as either line dispersion $\left(\sigma_{\mathrm{H} \beta}\right)$ or the full-width at halfmaximum intensity $\left(\mathrm{FWHM}_{\mathrm{H} \beta}\right)$ of $\mathrm{H} \beta$, and $\mathrm{L}$ is a monochromatic luminosity of AGN continuum at $5100 \AA$.

However, there are various sources of uncertainties in estimating BHM from single spectra, and the overall uncertainty of the SE mass estimates is not well quantified. The largest uncertainty comes from the unknown virial factor. An average virial factor has been determined by matching the $\mathrm{BH}$ mass-galaxy velocity dispersion $\left(\mathrm{M}_{\mathrm{BH}}-\sigma_{*}\right)$ relations of non-AGN and AGN samples, assuming that AGN host galaxies follow the same $\mathrm{M}_{\mathrm{BH}}-\sigma_{*}$ relation as non-AGN galaxies (Onken et al. 2004; Woo et al. 2010). However the virial factor of individual AGN may be different from this mean value. Thus, using an average virial factor can introduce random uncertainty in the mass estimates. Second, variability introduces random uncertainty on the SE mass estimates since both line width and continuum luminosity will be slightly different at different epochs. Third, the uncertainty and intrinsic scatter of the size-luminosity relation introduces random uncertainty.

Systematic uncertainty of SE mass estimates due to the difference of the line profiles between single spectra and the root-mean-spectra (rms) can be also significant as previous studies showed that the $\mathrm{H} \beta$ line widths are $\sim 20 \%$ broader in the mean spectra than in the rms spectra, indicating that SE masses can be systematically larger than reverberation masses (e.g., Collin et al. 2006).

In this paper, we present the main results from Park et al. (2011), including the uncertainties of BHM due to the variability, the overall uncertainty of SE method, and the systematic difference of line profiles, using 9 local Seyfert galaxies from the Lick AGN monitoring project (LAMP; Bentz et al. 2009b).

\section{Data reduction and measurements}

Using the homogeneous and high-quality spectra from the LAMP, we estimate multiple SE masses as well as BHMs from high quality mean and rms spectra for 9 local Seyfert 1 galaxies selected from the LAMP sample. To measure the line width of $\mathrm{H} \beta$ and the continuum luminosity at $5100 \AA$, we used the multi-component spectral fitting processes in a simultaneous and automated fashion. Detailed description of multi-component fitting process can be found in Park et al. (2011), and here we briefly summarize the method. 

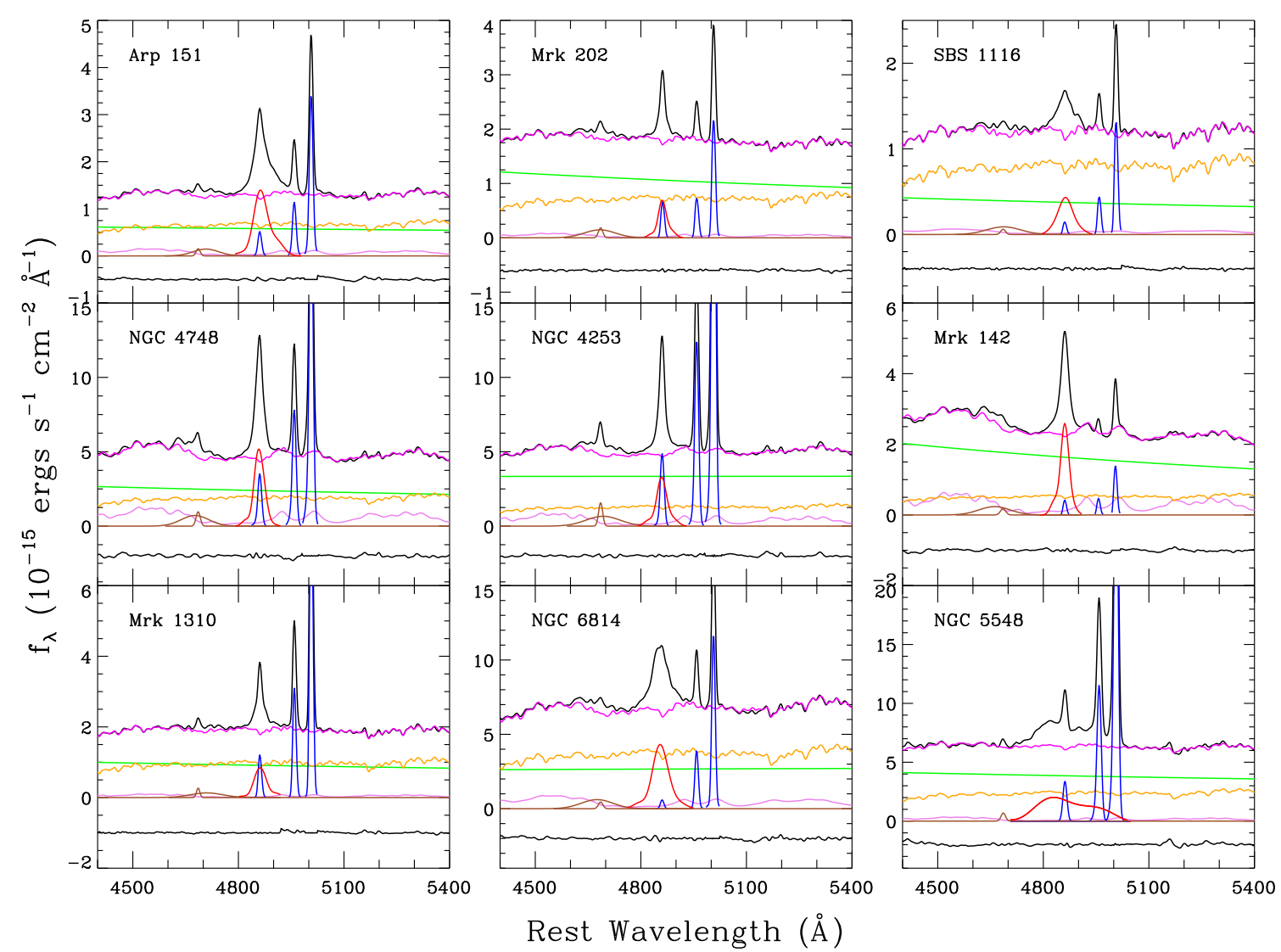

Figure 1: Multi-component spectral fitting results (Park et al. 2011). The mean spectra of all 9 Seyfert galaxies are presented along with multi-component models. In each panel, observed spectra (black) and the continuum $+[\mathrm{FeII}]+$ stellar best-fit model (magenta) are shown in the upper part, the best-fit power-law continuum (green), stellar absorption (yellow), and [FeII] template (violet) models are presented in the middle part. Three narrow lines, i.e., $\mathrm{H} \beta$, [OIII] $\lambda \lambda 4959,5007$ (blue), broad $\mathrm{H} \beta$ (red), and the broad and narrow $[\mathrm{HeII}] \lambda 4686$ components (brown) are presented in the bottom part. The residuals (black), representing the difference between the observed spectra and the sum of the all model components, are arbitrarily shifted downward for clarity.

First, we model the observed continuum with 3 components: the featureless AGN continuum, the Fe II emission blends, and the host galaxy starlight, using a single power-law continuum, a Fe II template from Boroson \& Green (1992), and a host-galaxy template from Bruzual \& Charlot (2003), which are convolved with various Gaussian velocity. Then, we subtract the narrow [OIII] lines, the narrow and broad components of [HeII] lines at $\lambda 4686$. Figure 1 presents the mean spectra of all 9 Seyfert galaxies and best fit models.

Once we remove all other components, we fit the broad component of the $\mathrm{H} \beta$ line with a Gauss-Hermite function and measure the line width. For AGN luminosity, we average the flux around $5100 \AA$ using the FeII and host galaxy starlight subtracted continuum. 

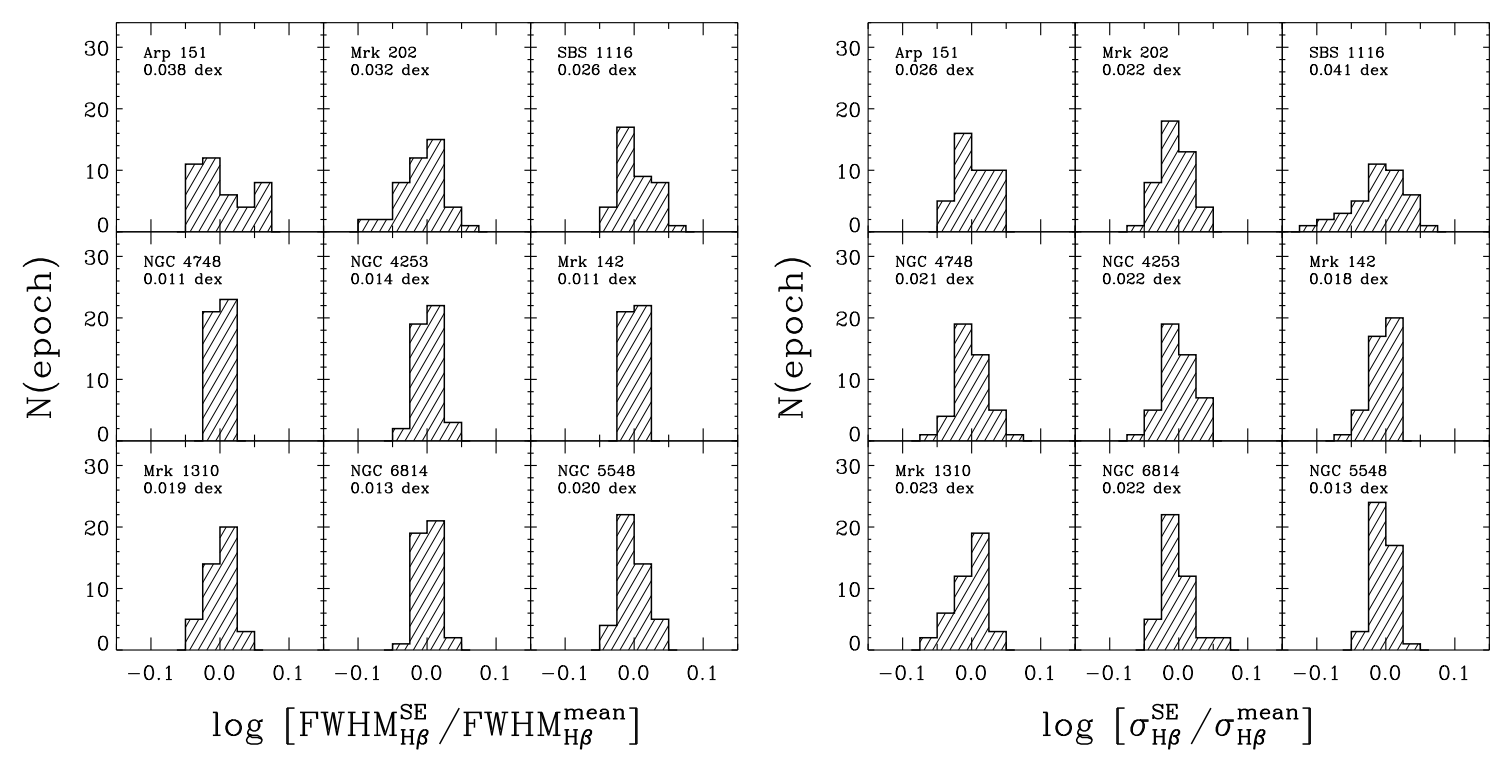

Figure 2: Left: Distribution of $\mathrm{FWHM}_{\mathrm{H} \beta}$ of $\mathrm{H} \beta$ measured from all single-epoch spectra (Park et al. 2011). Each $\mathrm{FWHM}_{\mathrm{H} \beta}$ value is normalized to the $\mathrm{FWHM}_{\mathrm{H} \beta}$ measured from the mean spectra. The average rms dispersion of 9 objects is $0.020 \pm 0.009$ dex. Right: Distribution of $\sigma_{\mathrm{H} \beta}$ of $\mathrm{H} \beta$. The average rms dispersion of 9 objects is $0.023 \pm 0.008$ dex.

\section{Analysis}

Using the measurements of the $\mathrm{H} \beta$ line width and AGN luminosity from each single-epoch spectra, we quantify the random uncertainty by investigating the distribution of the measurements. First, we compare line width and luminosity measurements, respectively. Then, we investigate the distribution of the virial products to study random error due to the combined effect.

\subsection{Uncertainties due to the line width variability}

First, we quantify the dispersion of the distribution of line width measurements using all SE spectra. This dispersion represents the random error due to the line width variability if we assume the measurement from the mean spectrum as a true value. In Figure 2, we present the distributions of $\mathrm{FWHM}_{\mathrm{H} \beta}$ and $\sigma_{\mathrm{H} \beta}$ measurements from all single-epoch spectra. All single-epoch values are normalized to the FWHM measured from the mean spectra. Thus, the distribution shows how much the line width can be randomly different compared to the mean value. The dispersion of FWHM distributions of individual objects ranges from 0.011 dex to 0.038 dex, with an average $0.020 \pm 0.009 \mathrm{dex}(\sim 5 \%)$. The amount of line width variation will introduce the uncertainty of the single-epoch $\mathrm{M}_{\mathrm{BH}}$ estimates by a factor of 2 in $\log$ scale, which is 0.040 dex. In Figure 2 we also show the distributions of line dispersion (right), which ranges from 0.013 dex to 0.041 dex, with an average and rms of $0.023 \pm 0.008 \mathrm{dex}(\sim 5 \%)$.

By averaging the dispersions of the distribution of the line width measurements for all 9 objects, we estimate the uncertainty of SE mass estimates due to the line width variation is on average 0.043 dex. Note that the dispersion of the line width distribution strongly depends on the vari- 


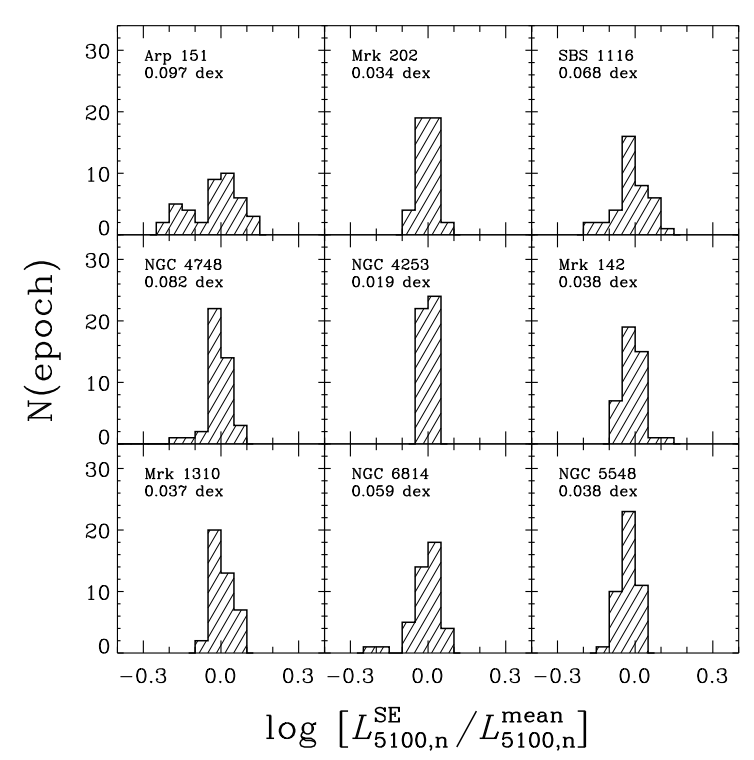

Figure 3: Distribution of the nuclear luminosities measured from spectra decompostion of each singleepoch spectra (Park et al. 2011). Each luminosity value is normalized to the luminosity measured from the mean spectra. The rms dispersion is given in each panel. The average rms dispersion of all 9 objects is $0.052 \pm 0.026$ dex.

ability. For example, Arp 151 with the highest variability amplitude shows the largest dispersion. Based on the these results, we conclude that the typical uncertainty of SE mass estimates due to the line width variability is $\sim 10 \%$.

\subsection{Uncertainties due to the luminosity variability}

AGN variability causes the variation of continuum luminosity as well, so here we test luminosity effect on SE mass estimates. In Figure 3, we present the distributions of the continuum luminosities at $5100 \AA$, after normalizing them by the luminosity measured from the mean spectra. Since the starlight contribution decreases the luminosity variability, we use the nuclear continuum luminosity $\left(\mathrm{L}_{5100, \mathrm{n}}\right)$, measured from each single spectrum based on the spectral decompositon. The dispersions of the luminosity distributions range from 0.019 to 0.097 dex, with an average $0.052 \pm 0.026 \mathrm{dex}(\sim 13 \%)$, which can be treated as a random error of the the continuum luminosity measurements from a single-epoch spectrum due to the luminosity variability.

Based on the virial assumption and the empirical size-luminosity relation, the random errors of the luminosity transfer to the uncertainty of the SE mass estimates by a $1 / 2$ power, which is 0.026 dex. This is somewhat smaller than the uncertainty of SE mass estimates due to the line width variability, $0.046 \mathrm{dex}$, as determined in the previous section.

\subsection{Uncertainties due to the combined variability}

Since the luminosity and the line width are anti-correlated as $V^{2} \propto L^{-0.5}$, one may naively expect that the variabilities of luminosity and line width can cancel out. However, two effects do not compensate each other since there is time lag between continuum and emission line variability and since the size-luminosity relation has non-negligible scatter. To quantify the combined effect 

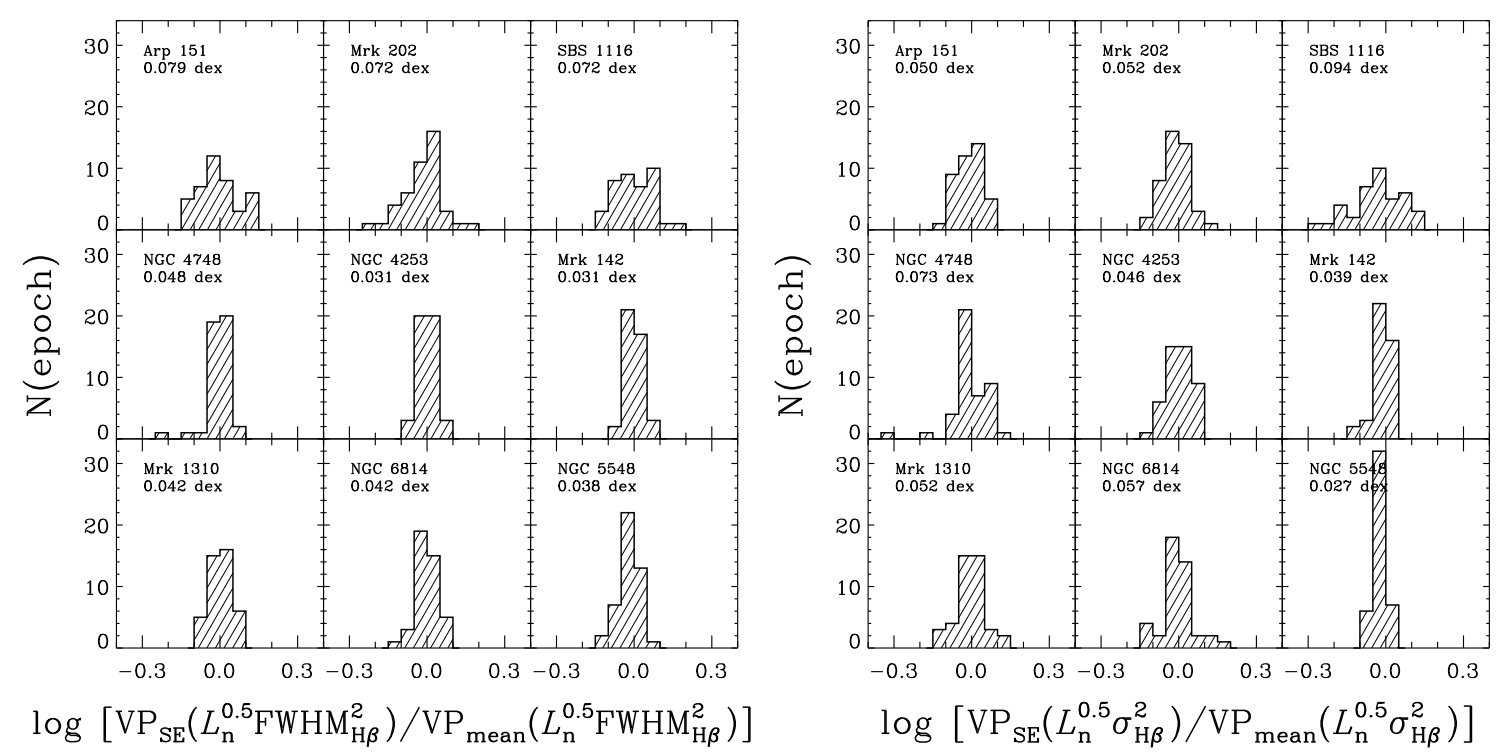

Figure 4: Distribution of the single-epoch virial product $\left(V^{2} \times L^{0.5}\right)$ normalized to that of the mean spectra (Park et al. 2011). Left: H $\beta$ line dispersion is used for the velocity and the nuclear luminosity at $5100 \AA$, corrected for the host galaxy, is used for the luminosity. The average rms dispersion of all 9 objects is $0.054 \pm 0.019$ dex. Right: $\mathrm{FWHM}_{\mathrm{H} \beta}$ is used for the velocity in calculating virial products. The average rms dispersion of all 9 objects is $0.051 \pm 0.019$ dex.

of variabilities of the continuum luminosity and line width, we investigate the distribution of the virial products $\left(L_{5100, n}^{0.5} \times \sigma_{H \beta}^{2}\right)$ measured from the SE spectra.

In Figure 4, we present the distributions of the SE virial products, after normalizing them by the virial product measured from the mean spectra. The dispersion of the distributions can be treated as a random error due to the combined variability. Left panel shows the virial products based on the line dispersion while right panel presents the virial products based on the $\mathrm{FWHM}_{\mathrm{H} \beta}$. The average random error of the virial products due to the combined variability is $0.054 \pm 0.019 \mathrm{dex}$ when the line dispersions $\left(\sigma_{H \beta}\right)$ are used, and $0.051 \pm 0.019$ dex when $\mathrm{FWHM}_{\mathrm{H} \beta}$ are used. We also calculate the dispersion of the virial product distribution, using the nuclear luminosity determined using constant host galaxy fraction from the mean spectra. In this case, the average dispersion is $\sim 0.10$ dex when the line dispersions $\left(\sigma_{H \beta}\right)$ are used, and $\sim 0.13$ dex when $\mathrm{FWHM}_{\mathrm{H} \beta}$ are used.

These results are consistent with previous studies (Wilhite et al. 2007, Woo et al. 2007, Denney et al. 2009). Thus, SE masses based on the spectra taken at different epochs are consistent within $\sim 30 \%$ uncertainty.

\subsection{Systematic difference of line width between rms and mean spectra}

To investigate the systematic differecne of $\mathrm{H} \beta$ between rms and mean spectra, we compare the broad $\mathrm{H} \beta$ line profiles from mean and rms spectra respectively. Generally the $\mathrm{H} \beta$ line is broader in the mean spectra than in the rms spectra. We compare the ratios of the line width measured from the mean spectra to those measured from the rms spectra as a function of line width. The average offset of $\mathrm{FWHM}_{\mathrm{H} \beta}$ is $0.08 \pm 0.04 \mathrm{dex}$. In the case of line dispersion $\left(\sigma_{H \beta}\right)$, the offset is slightly 
larger, $0.11 \pm 0.03$ dex. The larger offset of the line dispersion than FWHM demonstrates that the line shape is more different in the wings than in the core since the line dispersions are more affected by the wings.

There seems to be a systematic trend that the offset becomes larger for the narrower-line object. It is not clear why this systematic offset is present. In particular, NGC 4253 with the narrowest $\mathrm{H} \beta$ line width in the sample, shows the largest systematic difference. Perhaps, the systematic difference may be amplified due to the imperfect subtraction of narrow $\mathrm{H} \beta$, FeII blends, and stellar absorption, for this particular object has a very narrow $\mathrm{H} \beta$ line width $\left(<500 \mathrm{~km} \mathrm{~s}^{-1}\right)$.

\section{Discussion and conclusions}

There are 3 main sources of uncertianty in estimaing SE mass: 1) the uncertainty of the virial factor, 2) the random error due to the variability, and 3) the scatter of the size-luminosity relation. By taking the intrinsic scatter of the $\mathrm{AGN}_{\mathrm{BH}}-\sigma_{*}$ relation (Woo et al. 2010) as an upper limit of the uncertainty of the virial factor, we can assume 0.43 dex uncertainty due to the virial factor. Combining the virial factor uncertainty with 0.1 dex random error due to the variability and 0.13 dex scatter from the size-luminosity relation in quadrature, we estimate the the overall uncertainty of SE mass estimates as a factor of 3 .

The systematically larger $\mathrm{H} \beta$ line width in SE spectra than in rms spectra implies that SE masses can be overestimated if the same virial factor is used. For most obervational studies on BH-galaxy scaling relation evolution, this bias can introduce overestimation of BHM. This bias is significant for narrower line objects (e.g., narrow-line Seyfert 1 galaxies), implying that BHM of narrow line Seyfert 1 galaxies can be overestimated if the same virial factor is used. In constrast, the bias is not significant for massive $\left(>10^{7} \mathrm{M}_{\odot}\right)$ broad-line QSOs. Correcting for the bias found this study can slightly decrease $(\sim 25 \%)$ the amount of offset from the local scaling relation.

\section{Acknowledgments.}

This work has been supported by the Basic Science Research Program through the National Research Foundation of Korea funded by the Ministry of Education, Science and Technology (2010-0021558). We thank all LAMP collaborators for their suggestions and comments.

\section{References}

[1] Bentz, M. C., Peterson, B. M., Pogge, R. W., Vestergaard, M., \& Onken, C. A. 2006, ApJ, 644, 133

[2] Bentz, M. C., et al. 2009a, ApJ, 697, 160

[3] Bentz, M. C., et al. 2009b, ApJ, 705, 199

[4] Boroson, T. A., \& Green, R. F. 1992, ApJS, 80, 109

[5] Bruzual, G., \& Charlot, S. 2003, MNRAS, 344, 1000

[6] Collin, S., Kawaguchi, T., Peterson, B. M., \& Vestergaard, M. 2006, A\&AP, 456, 75

[7] Denney, K. D., et al. 2009, ApJ, 692, 246

[8] Denney, K. D., et al. 2010, ApJ, 721, 715 
[9] Kaspi, S., Maoz, D., Netzer, H., Peterson, B. M., Vestergaard, M., \& Jannuzi, B. T. 2005, ApJ, 629, 61

[10] Kaspi, S., Smith, P. S., Netzer, H., Maoz, D., Jannuzi, B. T., \& Giveon, U. 2000, ApJ, 533, 631

[11] Onken, C. A., Ferrarese, L., Merritt, D., Peterson, B. M., Pogge, R. W., Vestergaard, M., \& Wandel, A. 2004, ApJ, 615, 645

[12] Park, D., Woo, J.-H. et al. 2011, ApJ, submitted.

[13] Peterson, B. M., et al. 2004, ApJ, 613, 682

[14] Shen, Y., Greene, J. E., Strauss, M. A., Richards, G. T., \& Schneider, D. P. 2008, ApJ, 680, 169

[15] Wilhite, B. C., Brunner, R. J., Schneider, D. P., \& Vanden Berk, D. E. 2007, ApJ, 669, 791

[16] Woo, J.-H., \& Urry, M., 2002, ApJ, 579, 530

[17] Woo, J.-H., Treu, T., Malkan, M. A., \& Blandford, R. D. 2006, ApJ, 645, 900

[18] Woo, J.-H., Treu, T., Malkan, M. A., Ferry, M. A., \& Misch, T. 2007, ApJ, 661, 60

[19] Woo, J.-H., et al. 2010, ApJ, 716, 269 